\title{
Shape Optimization to Minimize The Response Time of Direct-acting Solenoid Valve
}

\author{
Yujeong Shin ${ }^{1}$, Seunghwan Lee ${ }^{2}$, Changhwan $\mathrm{Choi}^{3}$, and Jinho Kim ${ }^{1 *}$ \\ ${ }^{1}$ Department of Mechanical Engineering, Yeungnam University, Gyeongsan, Gyeongbuk 712-749, Korea. \\ ${ }^{2}$ Department of Mechanical Engineering, University of Michigan, Ann Arbor, MI 48105, USA \\ ${ }^{3}$ Gyeongbuk Institute for Advancement of Eco-friendly Auto Part Technology, Gyeongsan, Gyeongbuk 712-210, Korea
}

(Received 19 May 2015, Received in final form 15 June 2015, Accepted 15 June 2015)

\begin{abstract}
Direct-acting solenoid valves are used in the automotive industry due to their simple structure and quick response in controlling the flow of fluid. We performed an optimization study of response time in order to improve the dynamic performance of a direct-acting solenoid valve. For the optimal design process, we used the commercial optimization software PIAnO, which provides various tools for efficient optimization including design of experiments (DOE), approximation techniques, and a design optimization algorithm. 35 sampling points of computational experiments are performed to find the optimum values of the design variables. In all cases, ANSYS Maxwell electromagnetic analysis software was used to model the electromagnetic dynamics. An approximate model generated from the electromagnetic analysis was estimated and used for the optimization. The best optimization model was selected using the verified approximation model called the Kriging model, and an optimization algorithm called the progressive quadratic response surface method (PQRSM).
\end{abstract}

Keywords : solenoid valve, shape optimization, response time, design of experiments

\section{Introduction}

Satisfaction of environmental restrictions on the exhaust gases of automotives is required for car sales in Europe and the United States. The restrictions mainly result from $C O, H C$ and $N O_{x}$ due to the imperfect combustion of the engine $[1,2]$. In order to reduce these hazardous substances, providing exact ratio of fuel mixture is important prior to combustion [3]. The supply of fuel is determined by controlling a solenoid valve. The dynamic performance of direct-acting solenoid valves has been studied since the late 1990s. However, since the so-called "green car" is globally under the spotlight, the dynamic performance of direct-acting solenoid valves is gaining more attention, and related research is actively being conducted [4].

G. Tao et al. focused on the optimal design of a magnetic field for the high-speed response of a solenoid valve. They employed an axis symmetric plane simplified model for the simulation of the magnetic field. The optimum design was achieved through changes in parameters and the selection of solenoid materials. They

(C)The Korean Magnetics Society. All rights reserved.

*Corresponding author: Tel: +82-53-810-2441

Fax: +82-53-810-4627, e-mail: jinho@ynu.ac.kr claimed that the changes, produced greater magnetic force at lower power [5].

L. C. Passarini et al. studied the effect of the armature mass on dynamic response. They used the mass-springdamper system (MKsB) root-locus model to reduce the mass of the armature. This mass reduction improved the performance of electromagnetic fuel injectors [6].

Qilei Wang et al. used Al-Fe soft magnetic materials to re-design the structure of a magnetic circuit. This application enabled a more dense magnetic flux in the air gap of the valve in a short period of time, resulting in the reduction of the magnetic resistance [7].

These studies focused on individual performance improvements of the magnetic system or mechanical system of the valve. However, an overall optimization process covering the mechanical, electrical, and magnetic systems should be considered in order to maximize the dynamic performance, because the individual systems are coupled to each other. Hence, in this study, we conducted a dynamic performance improvement optimization for a direct-acting solenoid valve that enables the correct fuel amount by ensuring a response minimized through the entire shape optimization.

Factors that determine the response of a direct-acting solenoid valve are the shape of the yoke, and the coil and 
armature shape, as shown in Fig. 1. The number of turns in the coil and the cross sectional area between yoke and armature should be increased for stronger electromagnetic force, resulting in a faster response time. However the increase of the number of turns of the coil also causes a slow rising time of the current. Therefore, a tradeoff is required for the optimal design. Moreover, the coil and yoke size must be carefully designed due to the limited space available $[8,9]$. For the optimal design process, we used the commercial optimization software PIAnO (Process Integration and Design Optimization), which provides the design of experiment (DOE), approximation, and optimization functions (Ver.3.5.0.74, PIDOTECH, South Korea). Four design variables were selected, and an orthogonal array was used for the optimal design of the direct-acting solenoid valve. A commercial electromagnetic analysis program, ANSYS Maxwell was used to model the electromagnetic dynamics induced by the proposed design parameters in each case. (Ver.16. ANSOFT, USA) The approximate model was generated from the analysis of the response speed in each case. The geometry optimization was performed using the progressive quadratic response surface method (PQRSM) optimization algorithm.

\section{Structure and Operating Principle of a Direct-Acting Solenoid Valve}

The solenoid valve for the fuel pump is a direct-acting solenoid type. Figure 1 shows a structural diagram of the direct-acting solenoid valve. The valve is comprised of a coil, yoke, armature, and spring. The coil generates electromagnetic flux, and the yoke provides the pathway for the flux. The armature acts like a mechanical door for the on/off operation of the solenoid valve. The spring enables the armature to tick back to its initial position

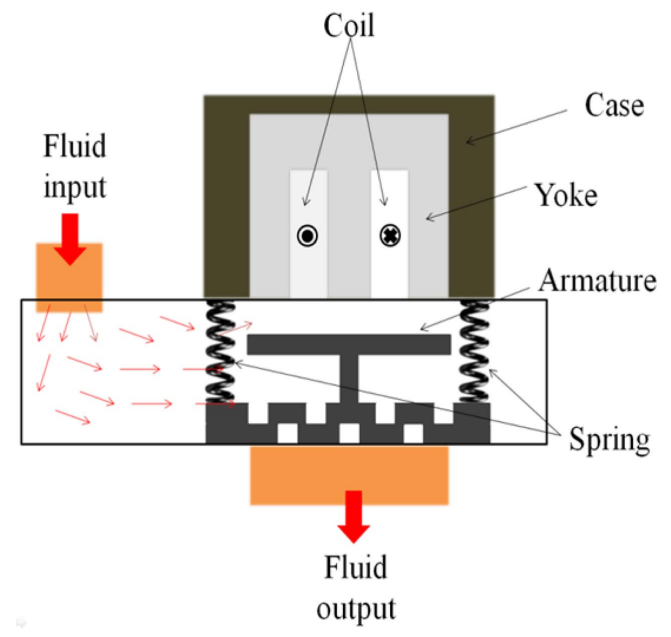

Fig. 1. (Color online) Structure diagram of a direct-acting solenoid valve.

when the voltage is removed. A direct-acting solenoid valve has a fast response time and a short stroke [10].

When voltage is supplied at the coil, an attractive force is induced between the yoke and the armature. The solenoid valve operates by this attractive force. The force is related to the rate of change of the energy in the electromagnetic field. Equation (1) is the governing equation of the mechanical system, including the force exerted between the air gap of the solenoid valves. External force is expressed as the sum of the pre-load $\left(F_{\text {pre-load }}\right)$, the spring force $\left(F_{\text {spring }}\right)$, and the electromagnetic force $\left(F_{\text {magnetic }}\right)$. Equation (2) describes the magnetic force in the air gap and is expressed a function of the position of the magnetic force that acts between the yoke and armature of the solenoid valve, where $\Phi$ is the magnetic flux and $R$ is the reluctance. The force relationship is dependent on the change in the reluctance of the system.

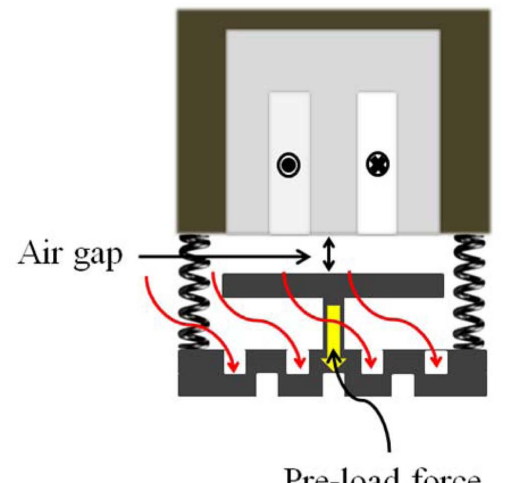

(a)

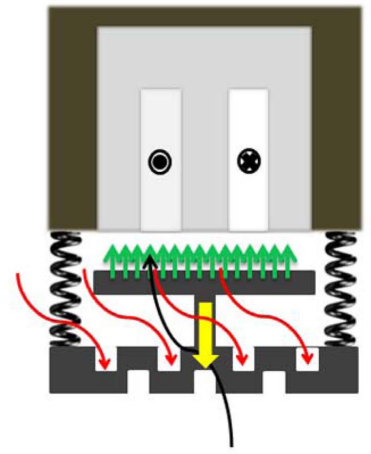

Electromagnetic force

(b)

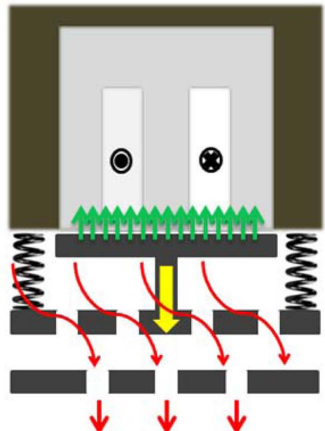

(c)

Fig. 2. (Color online) Principle of operation (a) at the lower end, (b) at transient position between upper and lower end, and (c) the upper end. 
When the voltage applied to the coil of the solenoid valve disappears, $F_{\text {magnetic }}$ becomes 0 . Then, the vibrator is returned to the initial state due to the restoring force of the spring. $B_{g}$ is the magnetic flux density in the air gap, $A_{g}$ is the cross section of air gap, and $\mu$ is permeability of air, as shown in Eq. (3).

$$
\begin{aligned}
& m \ddot{x}+c \dot{x}+k x=F_{\text {magnetic }}-F_{\text {pre-load }} \\
& F_{\text {manetic }}=-\frac{1}{2} \Phi^{2} \frac{d R}{d x} \\
& \Phi=B_{g} A_{g}, \frac{d R}{d x}=\frac{1}{\mu A_{g}}
\end{aligned}
$$

\section{Design Problem Definition}

\subsection{Design parameters for the response time of the solenoid valve}

The solenoid valve consists of an electromagnetic system and a mechanical system. A strong attractive force is required for the mechanical system. There are two ways to generate enough force: the flux must be increased, or the stroke should be decreased, as shown in Eq. (2). However, the stroke distance is fixed by the design requirement. Therefore, the flux should be increased to generate the stronger force. Equation (4) describes the magnetic flux density due to the solenoid, where $H_{z}$ is the magnetic flux, $i$ is the current flowing through the coil, and $n$ is the number of turns per unit length:

$$
B=\mu H_{z}, H_{z}=i n
$$

Therefore, the number of turns of the coil should be increased for a stronger magnetic force based on Eq. (4). However, increasing the turns of the coil causes a longer response time. This is indicated in Eq. (5), which shows the electrical system.

$$
\begin{aligned}
& V=i R+L \frac{d i}{d t}+K_{g} \frac{d x}{d t} \\
& L=N \frac{d \Phi}{d i}
\end{aligned}
$$

The relationship between the inductance and the magnetic flux rate, depending on the current, is expressed by Eq. (6), where $N$ is the number of coil turns, and $d \Phi / d i$ is the differential value of the magnetic flux depending on the current. Therefore, an increase in the number of coil turns results in a lower current rising speed. This means that the response time increases. A tradeoff between a sufficient attractive force and the number of coil turns is required, and this is why optimization is required for the design of the solenoid valve. In order to optimize the

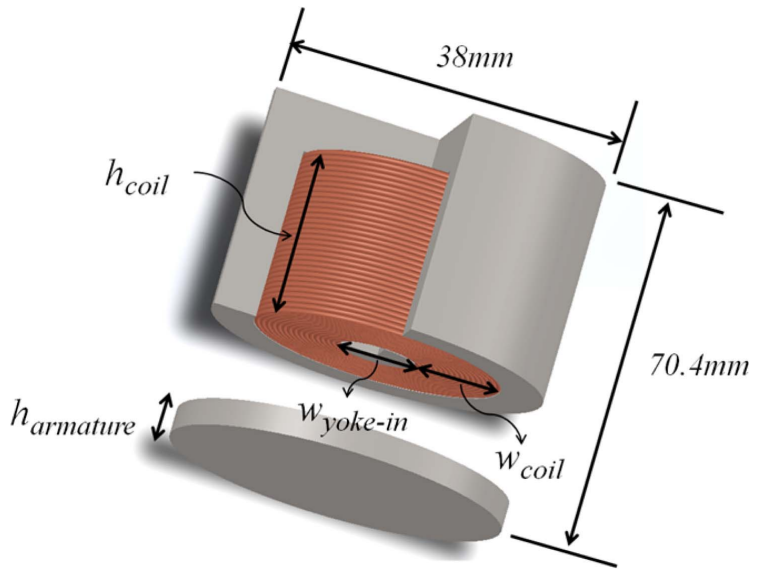

Fig. 3 (Color online) 3-D design variables for the solenoid valve.

solenoid valve, the design of the yoke, the number of coil turns, and the armature shape are considered because the yoke shape and number of coil turns determine the cross section of the air gap and the electromagnetic flux [11, 12].

Therefore, we designed the solenoid valve with a fixed size. The shape of the direct-acting solenoid valves was expressed with three-dimensional (3-D) design variable, as shown in Fig. 3. $w_{\text {yoke-in }}$ and $w_{\text {coil }}$ are independent design parameters; $w_{\text {yoke-out }}$ is a dependent design parameter following in the $x$ axial direction; $h_{\text {coil }}, h_{\text {amature }}$ are independent design parameters; and $h_{\text {yoke }}$ is a dependent design parameter following in the $z$ axial direction.

\subsection{Design problem}

\subsubsection{Design requirements}

The minimum thickness of $w_{\text {yoke-in }}$ during the cutting process for solenoid valve production is $2 \mathrm{~mm}$. The thickness of the upper yoke and armature should be at least $10 \mathrm{~mm}$ for fastening. The ultimate goal of the design is to satisfy at least of magnetic force generated between the yoke and armature for the direct-acting solenoid valve to operate, and to minimize the response time. In order to operate the valve, the magnetic force between the yoke and armature should be at least $400 \mathrm{~N}$.

\subsubsection{Design constraints}

The internal temperature of the coil in the solenoid valve has a range between -20 and $100^{\circ} \mathrm{C}$ for operation. We can calculate the maximum current and minimum resistance from Onderdonk's equation, as follows [13]:

$$
33\left(\frac{I}{A}\right)^{2} s=\log \left(\frac{T_{m}-T_{a}}{234+T_{a}}+1\right)
$$




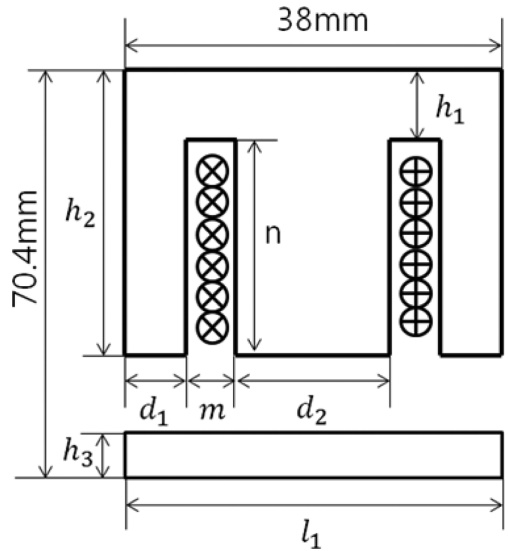

Fig. 4. 2-D design parameters of solenoid valve.

Since the direct-acting solenoid valve model has a minimum response time of $\sim 10 \mathrm{~ms}$, the maximum allowable current is considered under the assumption of an application time of $20 \mathrm{~ms}$. The conditions are as follows: $125^{\circ} \mathrm{C}$ ( $T_{m}$, enamel melting point), $100^{\circ} \mathrm{C}$ (maximum operating temperature), $0.2 \mathrm{~s}$ (application time), 1612 (circ. mils)(AWG standard \#18 coil cross-sectional area). When the input voltage is $24 \mathrm{~V}$, the maximum allowable current is $351 \mathrm{~A}$, based on Eq. (8). The minimum resistance is $0.07 \Omega$ from Eq. (9):

$$
\begin{aligned}
& R_{\text {coil }}=\frac{V}{I_{\max }}=\frac{24[\mathrm{~V}]}{351[\mathrm{~A}]}=0.07[\Omega] \\
& R_{\text {coil }} \geq 0.07[\Omega]
\end{aligned}
$$

\subsection{Design problem formulation}

Figure 4 shows the design parameters of the solenoid valve for the optimal design. We choose four design variables. When we compared Fig. 3 with Fig. 4, the design parameter $d_{2}$ is $w_{\text {yoke-in, }}, m$ is $w_{\text {coil }}, n$ is $h_{\text {coil }}$, and $h_{3}$ is $h_{\text {amature }}$. The initial value, lower limit, and upper limit for the design variables are summarized in Table 1. The design problem can be mathematically formulated as follows:

Table 1. Initial, lower, and upper limit values of the design variables selected.

\begin{tabular}{lcccc}
\hline \hline \multicolumn{2}{r}{ Design variables } & $\begin{array}{c}\text { Lower } \\
\text { bound }\end{array}$ & Initial & $\begin{array}{c}\text { Upper } \\
\text { bound }\end{array}$ \\
\hline$x_{1}(\mathrm{~mm})$ & $\frac{d_{2}}{2}$ & 4 & 7 & 10 \\
$x_{2}(\mathrm{~mm})$ & $m$ & 4 & 6 & 8 \\
$x_{3}(\mathrm{~mm})$ & $n$ & 21 & 30 & 39 \\
$x_{4}(\mathrm{~mm})$ & $h_{3}$ & 10 & 15 & 20 \\
\hline
\end{tabular}

Find $\frac{d_{2}}{2}, m, n, h_{3}$

To minimize response time

$$
\begin{aligned}
& \text { Subject to } d_{1}+\frac{d_{2}}{2}+m=19 \mathrm{~mm} \\
& h_{1}+h_{3}+n=70 \mathrm{~mm} \\
& 4 \mathrm{~mm} \leq \frac{d_{2}}{2} \leq 10 \mathrm{~mm} \\
& 4 \mathrm{~mm} \leq \mathrm{m} \leq 8 \mathrm{~mm} \\
& 21 \mathrm{~mm} \leq n \leq 39 \mathrm{~mm} \\
& 10 \mathrm{~mm} \leq h_{3} \leq 20 \mathrm{~mm} \\
& p m n \frac{\frac{d_{2}}{2}+2 m}{A_{\text {coil }}} \geq 0.07 \Omega\left(R_{\min }\right) \\
& F_{\text {max }} \geq 400 N
\end{aligned}
$$

\section{Optimal Design}

\subsection{Analysis procedures}

We generated a design of experiments (DOE) by applying the design constraints. The electromagnetic analysis using ANSYS Maxwell a commercial software, was performed according to sampling points of the DOE. Then a meta-model was generated using the Kriging algorithm provided by the PIAnO tool. After the Kriging model was generated, the design variables were modified in order to generate the approximation model. We used an optimization technique using the progressive quadratic response surface method (PQRSM) to find the optimum solution. Figure 5 shows the optimal analysis procedures.

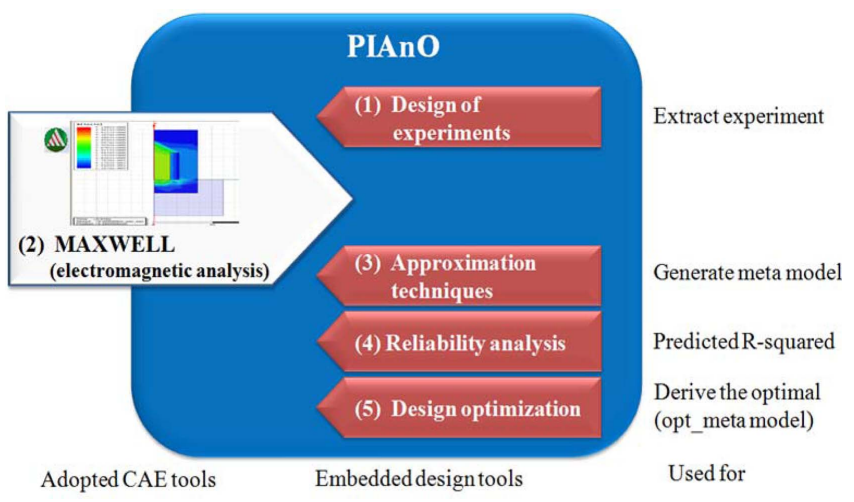

Fig. 5. (Color online) Diagram of optimal analysis procedures. 
Table 2. Layout and FEA results for L25 orthogonal array.

\begin{tabular}{|c|c|c|c|c|c|c|c|c|}
\hline No & $x_{1}(\mathrm{~mm})$ & $x_{2}(\mathrm{~mm})$ & $x_{3}(\mathrm{~mm})$ & $x_{4}(\mathrm{~mm})$ & $h_{1}(\mathrm{~mm})$ & $d_{1}(\mathrm{~mm})$ & Resistance $(\Omega)$ & $\begin{array}{l}\text { Response time } \\
(\mathrm{ms})\end{array}$ \\
\hline 1 & 4 & 4 & 21 & 10 & 39 & 11 & 0.07177 & $\mathrm{x}$ \\
\hline 2 & 4 & 5 & 25.5 & 15 & 29.5 & 10 & 0.118014 & $\mathrm{x}$ \\
\hline 3 & 4 & 6 & 30 & 17.5 & 22.5 & 9 & 0.179424 & $\mathrm{x}$ \\
\hline 4 & 4 & 7 & 34.5 & 20 & 15.5 & 8 & 0.257922 & $\mathrm{x}$ \\
\hline 5 & 4 & 8 & 39 & 12.5 & 18.5 & 7 & 0.35543 & $\mathrm{x}$ \\
\hline 6 & 5.5 & 4 & 25.5 & 12.5 & 32 & 9.5 & 0.108936 & $\mathrm{x}$ \\
\hline 7 & 5.5 & 5 & 30 & 20 & 20 & 8.5 & 0.17088 & $\mathrm{x}$ \\
\hline 8 & 5.5 & 6 & 39 & 10 & 21 & 7.5 & 0.283234 & $\mathrm{x}$ \\
\hline 9 & 5.5 & 7 & 21 & 17.5 & 31.5 & 6.5 & 0.188395 & $\mathrm{x}$ \\
\hline 10 & 5.5 & 8 & 34.5 & 15 & 20.5 & 5.5 & 0.373373 & $\mathrm{x}$ \\
\hline 11 & 7 & 4 & 30 & 15 & 25 & 8 & 0.153792 & 5.7 \\
\hline 12 & 7 & 5 & 39 & 17.5 & 13.5 & 7 & 0.263796 & $\mathrm{x}$ \\
\hline 13 & 7 & 6 & 34.5 & 12.5 & 23 & 6 & 0.294768 & 5.5 \\
\hline 14 & 7 & 7 & 25.5 & 10 & 34.5 & 5 & 0.266893 & 5.5 \\
\hline 15 & 7 & 8 & 21 & 20 & 29 & 4 & 0.263155 & 4.5 \\
\hline 16 & 8.5 & 4 & 34.5 & 17.5 & 18 & 6.5 & 0.206338 & 4.4 \\
\hline 17 & 8.5 & 5 & 21 & 12.5 & 36.5 & 5.5 & 0.164472 & 3 \\
\hline 18 & 8.5 & 6 & 25.5 & 20 & 24.5 & 4.5 & 0.250553 & 4.5 \\
\hline 19 & 8.5 & 7 & 39 & 15 & 16 & 3.5 & 0.466502 & 7 \\
\hline 20 & 8.5 & 8 & 30 & 10 & 30 & 2.5 & 0.4272 & 10 \\
\hline 21 & 10 & 4 & 39 & 20 & 11 & 5 & 0.266573 & 5 \\
\hline 22 & 10 & 5 & 34.5 & 10 & 25.5 & 4 & 0.30705 & 5.5 \\
\hline 23 & 10 & 6 & 21 & 15 & 34 & 3 & 0.233251 & 4 \\
\hline 24 & 10 & 7 & 30 & 12.5 & 27.5 & 2 & 0.403704 & 6.5 \\
\hline 25 & 10 & 8 & 25.5 & 17.5 & 27 & 1 & 0.406694 & 7 \\
\hline
\end{tabular}

\subsection{Design of experiments}

DOE was performed to increase the accuracy of the designed model. We used DOE to produce an approximation model that replaces the relationship between the factor and the characteristic value. And the approximation model explores the design area. Computational experiments were conducted following the orthogonal array shown in Table 2. After determining the experimental design using the orthogonal array provided by PIAnO, the direct-acting solenoid valve was designed according to each design variable. We were selected the orthogonal array by considering the number of saturated 4 points and 5 levels according to the number of design variables.

\subsection{Electromagnetic analysis}

Maxwell electromagnetic analysis software was used to model the electromagnetic behavior of the 25 experiments each model. 2-D cylindrical coordinates were used for the analysis, as shown in Fig. 6. The material used for the coil was copper, and the armature and core were made of steel 1010. The transient mode was selected to analyze the temporal behavior of the valve. We performed a

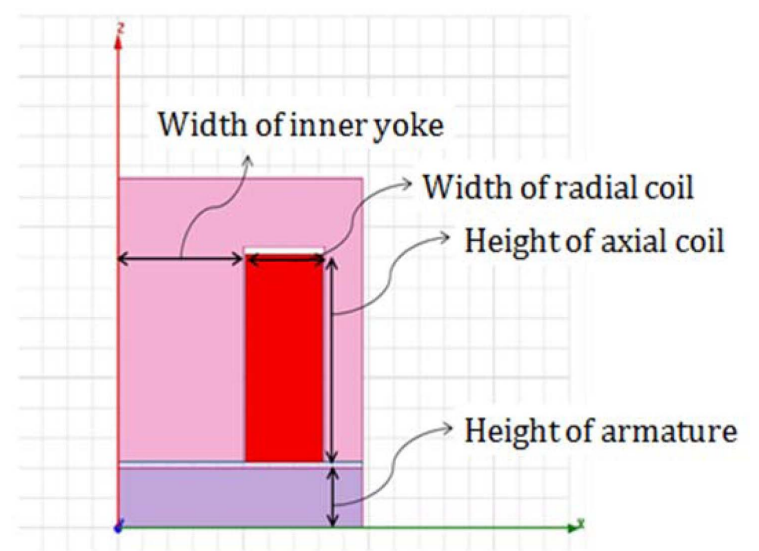

Fig. 6. (Color online) Maxwell 2-D model.

response time analysis of the solenoid valve using 25 sampling points, and the appropriate meta-model was produced from the results of the analysis.

\subsection{Approximation techniques}

A Kriging approximation model was produced based on 
Table 3. Layout and FEA results for L35 orthogonal array.

\begin{tabular}{|c|c|c|c|c|c|c|c|c|}
\hline No & $x_{1}(\mathrm{~mm})$ & $x_{2}(\mathrm{~mm})$ & $x_{3}(\mathrm{~mm})$ & $x_{4}(\mathrm{~mm})$ & $h_{1}(\mathrm{~mm})$ & $d_{1}(\mathrm{~mm})$ & Resistance $(\Omega)$ & $\begin{array}{c}\text { Response time } \\
(\mathrm{ms})\end{array}$ \\
\hline 1 & 7 & 3 & 20 & 10 & 40 & 9 & 0.072624 & 2 \\
\hline 2 & 7 & 4.25 & 25 & 15 & 30 & 7.75 & 0.138061 & 3.48 \\
\hline 3 & 7 & 5.5 & 30 & 17.5 & 22.5 & 6.5 & 0.229086 & 6.7 \\
\hline 4 & 7 & 6.75 & 35 & 20 & 15 & 5.25 & 0.349036 & 0 \\
\hline 5 & 7 & 8 & 40 & 12.5 & 17.5 & 4 & 0.501248 & 0 \\
\hline 6 & 7.75 & 3 & 25 & 12.5 & 32.5 & 8.25 & 0.09879 & 2.29 \\
\hline 7 & 7.75 & 4.25 & 30 & 20 & 20 & 7 & 0.179291 & 3.75 \\
\hline 8 & 7.75 & 5.5 & 40 & 10 & 20 & 5.75 & 0.328944 & 6.34 \\
\hline 9 & 7.75 & 6.75 & 20 & 17.5 & 32.5 & 4.5 & 0.213867 & 3.46 \\
\hline 10 & 7.75 & 8 & 35 & 15 & 20 & 3.25 & 0.468496 & 7.22 \\
\hline 11 & 8.5 & 3 & 30 & 15 & 25 & 7.5 & 0.12816 & 2.62 \\
\hline 12 & 8.5 & 4.25 & 40 & 17.5 & 12.5 & 6.25 & 0.25721 & 4.66 \\
\hline 13 & 8.5 & 5.5 & 35 & 12.5 & 22.5 & 5 & 0.308385 & 5.03 \\
\hline 14 & 8.5 & 6.75 & 25 & 10 & 35 & 3.75 & 0.285356 & 4.3 \\
\hline 15 & 8.5 & 8 & 20 & 20 & 30 & 2.5 & 0.2848 & 4 \\
\hline 16 & 9.25 & 3 & 35 & 17.5 & 17.5 & 6.75 & 0.160734 & 3.09 \\
\hline 17 & 9.25 & 4.25 & 20 & 12.5 & 37.5 & 5.5 & 0.137683 & 2.45 \\
\hline 18 & 9.25 & 5.5 & 25 & 20 & 25 & 4.25 & 0.23496 & 0 \\
\hline 19 & 9.25 & 6.75 & 40 & 15 & 15 & 3 & 0.485406 & 7.12 \\
\hline 20 & 9.25 & 8 & 30 & 10 & 30 & 1.75 & 0.452832 & 6.421 \\
\hline 21 & 10 & 3 & 40 & 20 & 10 & 6 & 0.196512 & 3.74 \\
\hline 22 & 10 & 4.25 & 35 & 10 & 25 & 4.75 & 0.256832 & 0 \\
\hline 23 & 10 & 5.5 & 20 & 15 & 35 & 3.5 & 0.199716 & 3.2 \\
\hline 24 & 10 & 6.75 & 30 & 12.5 & 27.5 & 2.25 & 0.385682 & 5.76 \\
\hline 25 & 10 & 8 & 25 & 17.5 & 27.5 & 1 & 0.39872 & 6.25 \\
\hline 26 & 7.15 & 6.75 & 29 & 11.5 & 29.5 & 5.1 & 0.29338227 & 5.86 \\
\hline 27 & 7.45 & 4.25 & 37 & 14.5 & 18.5 & 7.3 & 0.21440723 & 6.85 \\
\hline 28 & 7.75 & 5.75 & 21 & 13.5 & 35.5 & 5.5 & 0.18269475 & 3.18 \\
\hline 29 & 8.05 & 6.25 & 39 & 18.5 & 12.5 & 4.7 & 0.38788425 & 6.35 \\
\hline 30 & 8.35 & 7.75 & 25 & 15.5 & 29.5 & 2.9 & 0.33728775 & 4.77 \\
\hline 31 & 8.65 & 3.75 & 23 & 17.5 & 29.5 & 6.6 & 0.12926805 & 2.47 \\
\hline 32 & 8.95 & 7.25 & 33 & 19.5 & 17.5 & 2.8 & 0.42842019 & 6.19 \\
\hline 33 & 9.25 & 3.25 & 35 & 12.5 & 22.5 & 6.5 & 0.17615325 & 3.3 \\
\hline 34 & 9.55 & 4.75 & 27 & 10.5 & 32.5 & 4.7 & 0.21778389 & 3.64 \\
\hline 35 & 9.85 & 5.25 & 31 & 16.5 & 22.5 & 3.9 & 0.28911561 & 4.68 \\
\hline
\end{tabular}

the DOE simulation results. This method models the relationship between the value of the response time of the actual analysis using Maxwell and the design variables within a local or entire area of interest. The Kriging model, one of interpolation model, gives excellent predictive performance in nonlinear systems containing several design variables. The 25 computational experiments described in Section 3.1 were performed. The approximation model was evaluated and the $R^{2}$ is $52 \%$, which is low.

In order to improve the accuracy of the model, the magnetic force distribution of experimental point No. 1 and No. 11 were compared, as shown in Fig. 7. At the
No. 1 sampling point, the electromagnetic force acts from the center out to $4 \mathrm{~mm}$ in the radial direction, despite a 7 $\mathrm{mm}$ length of the yoke radius. Therefore, the magnetic force is not transmitted to the outer yoke and the displacement of the armature is zero. The corresponding response time of the armature does not exist. However, at the No. 11 sampling point, the electromagnetic force is evenly distributed up to $7 \mathrm{~mm}$ throughout the yoke, and the displacement of the armature is $0.74 \mathrm{~mm}$. The corresponding response time of the armature is $5.7 \mathrm{~s}$. Based on this result, the radius less than $7 \mathrm{~mm}$ has no movement of armature. Since the valve starts to operate above $7 \mathrm{~mm}$, the lower limit of $x_{1}$ was modified to $7 \mathrm{~mm}$. The modified 
Table 4. Stopping criteria of parameters of PQRSM.

\begin{tabular}{lc}
\hline \hline Maximum number of iterations & 40 \\
Maximum number of consecutive iterations & 2 \\
Violated constraint limit & 0.003 \\
Max. relative change in the objective function & 0.001 \\
Max. relative change in the design variables & 0.1 \\
\hline
\end{tabular}

initial values of the design variables, the lower limits, and the upper limits are summarized Table 5. Computational experiments of modified design parameters are summarized in Table 3.

\subsection{Design optimization}

We selected the progressive quadratic response surface method (PQRSM) of optimization technique on PIAnO because it is suitable for actual problem that requires the use of CAE Solver including numerical noise as shown in this paper. The PQRSM is a technique to find the optimal solution to the repeated use of a trust region and response surface model (RSM). The method performs $2 n+1$ simulations (where $\mathrm{n}$ is the number of design variables) and, produces a quadratic response surface model for the function of the constraints and objective function, and corresponds to a sequential approximate optimal design method for optimal design [14]. It is an advantage to be able to ensure robustness and efficient convergence at the same time by applying the intelligent Hessian update rule to improve the quality of the approximation model and controlling confidence intervals algorithm for adjusting the design area effectively [15]. The stopping criteria of parameters of PQRSM were set as shown in the Table 4. The maximum number of consecutive iterations of parameter of PQRSM was set up 2 times. This parameter means it will terminate the optimization if the objective function or constraints do not change during iteration two times.

\subsection{Optimal design results}

Kriging model accuracy was improved to $82 \%$ and the response time was reduced to $1.828 \mathrm{~ms}$ which is reduced to $\sim 60 \% \%$, compared with the initial value. In this study, we conducted an optimal design using an approximate model. So, the optimal design results can vary depending on the accuracy of the Kriging model [16]. The accuracy of optimized results should be verified by analysis using Maxwell. For this validation, the optimization of the Kriging model results (Opt_meta) of the optimal design variable and the analysis results from Maxwell (Opt_exact) were compared, as shown in Fig. 7.

The optimization of Kriging model results (Opt_meta)

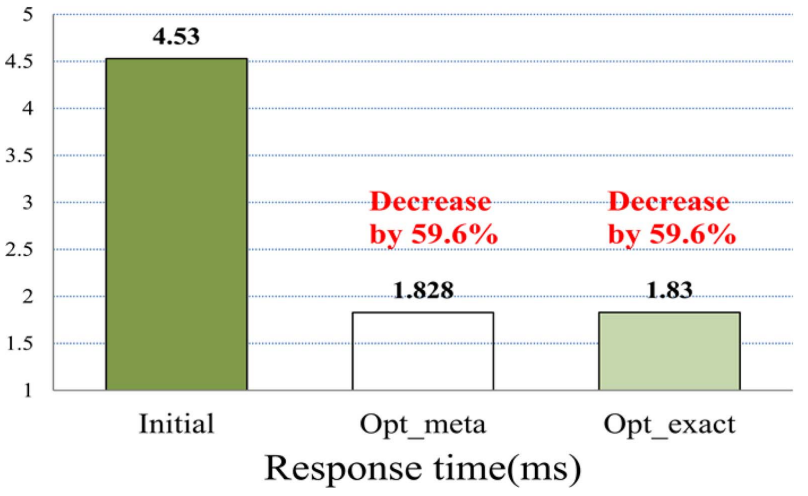

Fig. 7. (Color online) Comparison of the response time.

Table 5. Initial and optimal design variable values.

\begin{tabular}{lccccc}
\hline \hline \multicolumn{2}{c}{ Design variables } & $\begin{array}{c}\text { Lower } \\
\text { bound }\end{array}$ & Initial & Optimal & $\begin{array}{c}\text { Upper } \\
\text { bound }\end{array}$ \\
\hline$x_{1}(\mathrm{~mm})$ & $\frac{d_{2}}{2}$ & 4 & 7 & 7.75 & 10 \\
$x_{2}(\mathrm{~mm})$ & $m$ & 4 & 6 & 3 & 8 \\
$x_{3}(\mathrm{~mm})$ & $n$ & 21 & 30 & 20 & 39 \\
$x_{4}(\mathrm{~mm})$ & $h_{3}$ & 10 & 15 & 10 & 20 \\
\hline
\end{tabular}

of the optimal design variables and the analysis results from Maxwell (Opt_exact) are very similar with an error range of $0.1 \%$. Therefore, we confirmed the high accuracy of the Kriging model's prediction. The initial and optimal values for the design variables $\left(x_{1}, x_{2}, x_{3}, x_{4}\right)$ were compared as shown in Table 5. To sum up, the optimum values for the four design parameters are each $x_{1}\left(h_{2} / 2\right.$ : length of inside yoke) is $7.75 \mathrm{~mm}, x_{2}$ ( $m$ : length of coil) is $3 \mathrm{~mm}, x_{3}\left(n\right.$ : height of coil) is $20 \mathrm{~mm}, x_{4}\left(h_{3}\right.$ : height of armature) is $10 \mathrm{~mm}$. Compared to the shape of the initial model, the radius of the central core increased. However, the outside thickness of the core, the width of the coil, the height of the coil, and the height of the armature were reduced.

\section{Conclusion}

In this study, we conducted an optimal design in order to reduce the response time in a direct-acting solenoid valve.

1) The valve design problem was formulated under design requirements in terms of the external size of the initial solenoid valves.

2) We created an orthogonal array using commercial PIDO tools in PIAnO software. According to the sampling points that were analyzed with the commercial electromagnetic analysis program MAXWELL, the direct- 
acting solenoid valve was modeled to determine the response time.

3) Based on our simulation results according to the design of experiments, a Kriging model was generated using PIAnO. This model was used for the optimal design. As a result, we reduced the response time by $\sim 60 \%$ compared with the initial model. Moreover, we derived the shape of a solenoid valve having a $1.83 \mathrm{~ms}$ response time.

We suggest that the results of this study can be used to guide the optimization of parameters with respect to an overall optimization process. The proposed process covers the mechanical, electrical, and magnetic systems in order to achieve a fast response time to improve the dynamic performance of a direct-acting solenoid valve. In future work, a prototype will be produced using the optimal model, and the actual response time will be validated.

\section{References}

[1] P. W. Park, KSAE. 20, 11 (1998).

[2] C. W. Park, H. S. Kim, S. J. Woo, and Y. R. Kim, KSME. 36, 335, (2012).

[3] Aditya Mulemane, J. S. Han, P. H. Lu, S. J. Yoon, and M. C. Lai, KIMM. doi:10.4271/2004-01-0536 (2004).

[4] Ryan R. Chladny, Charles Robert Koch, and Alan F.
Lynch., IEEE Trans. Magn. 41, 1155 (2005).

[5] G. Tao, H. Y. Chen, Y. Y. J., and Z. B. He, J. of Materials Processing Technology 129, 555 (2002).

[6] L. C. Passarini and P. R. Nakajima, J. Braz. Soc. Mech. Sci. \& Eng. 25, 329 (2003).

[7] Q. Wang, Fengyu Yang, Q. Yang, Junhui Chen, and Hongyan Guan, Energy Conversion and Management 52, 2309 (2011).

[8] M. Taghizadeh, A. Ghaffari, and F. Najafi, Comptes Rendus Mecanique, 337, 131 (2009).

[9] Song June, Huang Jian-ping, Li Xiao-lu, Li Shu-ze, and Huang Zhen, Vehicle Engine 48 (2005).

[10] Qianfeng Liu., Hanliang Bo, and Benke Qin, Nuclear Engineering and Design. 240, 2890 (2010).

[11] J. H. Kim, Journal of the Korean Society for Precision Engineering 25, 53 (2008).

[12] C. C. Hwang and Y. H. Cho, IEEE Trans. Magn. 37, 3021 (2001).

[13] S. J. Lee, J. H. Kim, B. S. Song, and J. H. Kim, J. Magn. 18, 481 (2013).

[14] J. H. Choi, T. H. Kim, K. B. Jang, and J. Lee, IEEE Trans. Magn. 39, 3241 (2003).

[15] C. H. Park, H. J. Shim, D. H. Choi, J. K. Kim, and S. M. Lee, KSAE Conference 2264 (2009).

[16] K. B. Lee, C. H. Park, and J. H. Kim, Hindawi Publishing Corporation Advances in Mechanical Engineering, Article ID 545126 (2014). 